5CB $(\Delta \varepsilon>0)$, MBBA $(\Delta \varepsilon<0), \operatorname{MBBA}+5 \mathrm{CB}(\Delta \varepsilon=0)$

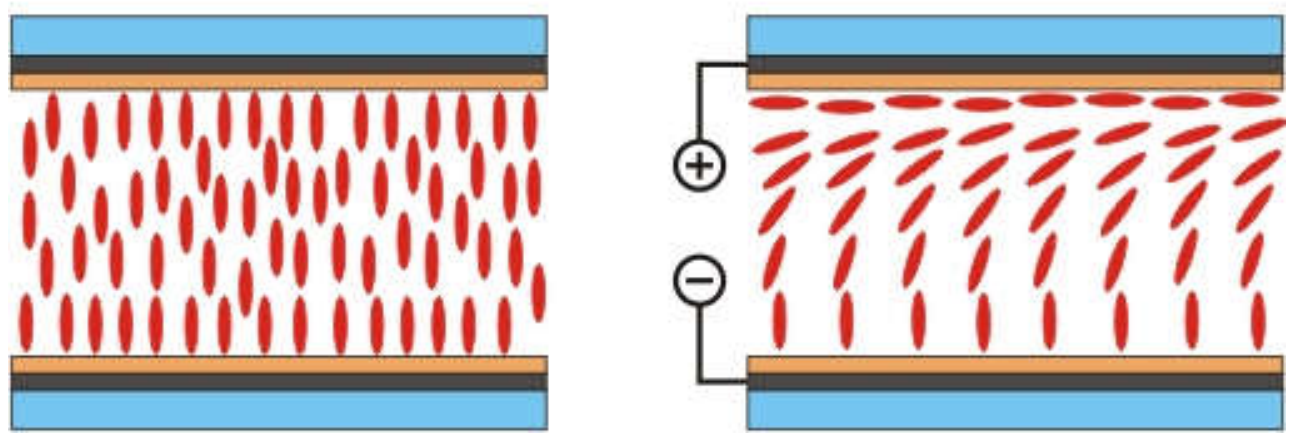


Electrically induced anchoring transition in nematics with small or zero dielectric anisotropy

Vitaly Sergeevich Sutormin ${ }^{\mathrm{a}}$, Mikhail Nikolaevich Krakhaleve, ${ }^{\mathrm{a}, \mathrm{b}}$, Oxana

Olegovna Prishchepa ${ }^{\mathrm{a}, \mathrm{b}}$ and Victor Yakovlevich Zyryanov ${ }^{\mathrm{a}}$

${ }^{a}$ Kirensky Institute of Physics, Krasnoyarsk Scientific Center, Siberian Branch, Russian Academy of Sciences, Krasnoyarsk 660036, Russia; ${ }^{b}$ Siberian Federal University, Krasnoyarsk 660041, Russia

Corresponding author: V.Ya. Zyryanov, e-mail: zyr@iph.krasn.ru 


\title{
Electrically induced anchoring transition in nematics with small or zero dielectric anisotropy
}

\author{
The orientational transitions induced by electrically controlled ionic modification \\ of surface anchoring in liquid crystal cells based on the nematics with small or \\ zero dielectric anisotropy $\Delta \varepsilon$ are considered. The type of director reorientation is \\ shown to be independent of the sign of dielectric anisotropy and can be the same \\ for the nematics with both negative and positive $\Delta \varepsilon$. Besides, the orientational \\ transition and corresponding switchable optical states do not depend on the $\Delta \varepsilon$ \\ value and can be effectively realized even for the nematics with zero dielectric \\ anisotropy.
}

Keywords: nematic, ionic surfactant, anchoring transition, hybrid homeoplanar structure, dielectric anisotropy, electrooptics

\section{Introduction}

At present, the cells consisting of two parallel substrates filled with liquid crystal (LC) are widely used in modern optoelectronic devices. The macroscopic optical properties of the cells are mainly specified by the LC orientational structure which is highly sensitive to external forces. In the commercially available LC devices the director reorientation and the corresponding switching of the optical states result from the Frederiks effect [1] under the action of electric field. The reorientation of director $\mathbf{n}$ is caused by the external electric field $\mathbf{E}$ which exerts the torque $\boldsymbol{\Gamma}$ on the polarization $\mathbf{P}$ of LC [2]:

$$
\boldsymbol{\Gamma}=\mathbf{P} \times \mathbf{E}=\varepsilon_{0} \Delta \varepsilon(\mathbf{E n}) \mathbf{n} \times \mathbf{E},
$$

where $\varepsilon_{0}$ is the dielectric constant and $\Delta \varepsilon$ is a dielectric anisotropy of LC. Equation (1) makes it obvious that the dielectric anisotropy is the principal parameter which determines the characteristics of the LC reorientation process, for example, the value of the Frederiks threshold field $E_{c}$ : 


$$
E_{c}=\pi \sqrt{K /\left(\varepsilon_{0} \Delta \varepsilon\right)} / d
$$

where $K$ is an appropriate LC elastic modulus depending on the type of orientational transition, $d$ is a gap thickness of the LC cell. Moreover, the design of LC cell is specified by the $\Delta \varepsilon$ sign. Figure 1 shows the Frederiks effect within LC cells based on the nematics with different dielectric anisotropy. The ac electric field is applied perpendicularly to the LC layer.

If the nematic has a positive dielectric anisotropy, LC molecules are reoriented along the electric field (Figure 1(a)). In the cell based on the nematic with negative dielectric anisotropy, the electric field induces the reorientation of LC molecules perpendicularly to the field (Figure 1(b)). Consequently, the director orientation must be homeotropic in the initial state. The equation (2) implies that $E_{c} \rightarrow \infty$ for LC with zero dielectric anisotropy. It means that the reorientation of LC with $\Delta \varepsilon=0$ caused by the Frederiks effect is principally impossible (Figure 1(c)). It should be noted that the director reorientation by means of the Frederiks effect occurs under the invariable anchoring of the LC molecules at the interface.

An alternative way to reorient LC is possible by modifying the LC surface anchoring with substrates by various external effects [3-11]. The methods of electrically induced modification of the surface anchoring seem to be the most interesting for practical application [8-11]. We are developing the method of electrically induced modification of surface anchoring of nematics by using ionic surfactants. The ionicsurfactant method was applied previously both to the polymer dispersed liquid crystals [12-15] and the LC layer cells [16-18].

In this article we investigate and compare the LC reorientation and corresponding optical response induced by the electrically controlled ionic modification 
of surface anchoring within LC cells based on the nematics with the different sign and value of dielectric anisotropy, including $\Delta \varepsilon=0$.

\section{Materials and experimental techniques}

The experiments were carried out with sandwich-like cells. These cells consisted of two glass substrates with transparent ITO electrodes coated with the $1.5 \mu \mathrm{m}$-thick polymer films and $10 \mu \mathrm{m}$-thick LC layer between them. The rubbed polymer films based on the polyvinyl alcohol (PVA) doped with glycerin compound (Gl) in the weight ratio PVA $: G 1=1: 0.29$ were used as orienting coatings. The utilized liquid crystal materials were the nematics LC 4-methoxybenzylidene-4'-n-butylaniline (MBBA) with $\Delta \varepsilon=$ 0.54 at $25^{\circ} \mathrm{C}[19]$ and the MBBA-5CB mixture in the weight ratio $1: 0.02$, respectively. 5CB is 4-n-pentyl-4'-cyanobiphenyl with $\Delta \varepsilon=+13.3$ at $25^{\circ} \mathrm{C}$ [20]. The MBBA-5CB mixture had the zero dielectric anisotropy [8] and that was confirmed by our measurements of dielectric permittivity. The nematics were preliminary doped with ionic surfactant cethyltrimethylammonium bromide $(\mathrm{CTAB})$ in the weight ratio $\mathrm{LC}: \mathrm{CTAB}=1: 0.008$. CTAB dissolved in liquid crystal dissociates into the positively charged surface-active ion $\mathrm{CTA}^{+}$and the negative ion $\mathrm{Br}^{-}$.

The optical textures of the nematic layer and their transformations under the electric field were studied in the crossed polarizers by means of the optical microscopy. Electro-optical response of the system consisting of LC cell placed between crossed polarizers was studied by using He-Ne laser $(\lambda=633 \mathrm{~nm})$. The laser beam passed sequentially through the polarizer, LC cell, analyzer and was detected by the photodiode. The LC cell was placed so that the angle between the substrate rubbing direction and the polarizer made $45^{\circ}$. The transmittance of the system was determined as $T=\left(I_{t} / I_{0}\right) \times 100 \%$, where $I_{0}$ is intensity of radiation after the first polarizer, $I_{t}$ is intensity after the second polarizer. 


\section{Results and discussion}

The polymer films specify the planar anchoring for the used nematics. However the homeotropic director orientation within cell is formed in the initial state due to the ionic surfactant addition in the LC. It can be explained by the formation of surface-active $\mathrm{CTA}^{+}$layers being adsorbed at the substrates. These layers screen the orienting influence of polymer walls and promote the LC molecules alignment perpendicularly to the substrates (Figure 2(a)). The application of dc electric field makes the electrodeanode substrate free from the layer of $\mathrm{CTA}^{+}$ions and the planar anchoring proper to the polymer film restored (Figure 2(b)). As a result, the transition from the homeotropic director configuration into the hybrid homeoplanar one occurs within the LC cell (Figure 2). This orientational transition has been investigated previously in the LC cell based on $5 \mathrm{CB}(\Delta \varepsilon>0)$ doped with $\mathrm{CTAB}[16,17]$.

Since the main factor influencing the anchoring transition is the movement of the surface-active ions, the same variant of the director reorientation can be realized for the different LC independently of the sign and value of their dielectric anisotropy (Figure 2). For instance, the transition from the homeotropic director configuration into the homeoplanar one caused by the ionic modification of surface anchoring begins at $U=2.7 \mathrm{~V}$ for the cell based on $\operatorname{MBBA}(\Delta \varepsilon<0)$ doped with CTAB. This value is close to the threshold voltage $\left(U_{t h}=2.8 \mathrm{~V}\right)$ of 5CB-cell operated by the ionic-surfactant method $[16,17]$. The reorientation of MBBA can not be explained by the Frederiks effect (Figure 1(b)) since the threshold field (Equation (2)) would be about $4 \mathrm{~V}$ for our cell [21]. Moreover, the estimation of phase retardation value reveals that it is approximately twice less than the one for the planar MBBA layer (Figure 1(b), right column) and corresponds to the hybrid configuration. The reorientation of LC director is not accompanied by the electrohydrodynamic instability in the range of the control voltage $2.7 \mathrm{~V} \leq U \leq 4 \mathrm{~V}$. The domain structure was formed at the control voltage 
$U \geq 4.1 \mathrm{~V}$. The on/off time of the MBBA-cell under the action of rectangular electric pulse is tens of seconds and exceeds the same parameters for the 5CB-cell ( 1 s) [17].

It should be emphasized that the analogous transformation of the orientational structure (Figure 2) occurs for the nematic with zero dielectric anisotropy. The figures 3(a) and 3(b) demonstrate the change of the optical texture of the LC cell based on the MBBA-5CB mixture $(\Delta \varepsilon=0)$ doped with $\mathrm{CTAB}$ and placed between the crossed polarizers under the action of dc electric field. In the initial state, the optical texture of the LC layer is a uniform dark area independently of the sample rotation on the microscopic stage (Figure 3(a)). It means that the director orientation within the cell is homeotropic. The dc electric field induces the modification of the surface anchoring which results in the formation of the hybrid LC structure. This transition leads to the increase of the light transmission of the system (Figure 3(b)). The reorientation induced by the ionic modification of the surface anchoring in the cell filled with MBBA-5CB mixture $(\Delta \varepsilon=0)$ has a threshold character starting from $3 \mathrm{~V}$ which is approximately equal to the threshold voltages of 5CB-cell $[16,17]$ and MBBA-cell operated by the ionic-surfactant method. At the control voltage $3.0 \mathrm{~V} \leq U \leq 4.1 \mathrm{~V}$ the optical texture of LC layer is a uniform light area (Figure 3(b)) without a domain structure which is formed at $U>4.1 \mathrm{~V}$. Thus the ionic-surfactant method provides an opportunity of the electrically controlled director reorientation of LC with zero dielectric anisotropy. The figure 3(c) shows the oscillogram of the square-wave response of the LC cell filled with the MBBA-5CB mixture and placed between crossed polarizers. The pulse amplitude is $4 \mathrm{~V}$ and its duration is $50 \mathrm{~s}$. In the initial state, the cell transmittance is close to zero because of the homeotropic director orientation within the LC cell. The nematic reorientation into the hybrid state makes the light transmission of the system 
increased and saturated at near $66 \%$ after having successively passed through the maximum and minimum.

The dynamical characteristics of the optical response of the LC cell based on the nematic with $\Delta \varepsilon=0$ depending on the value of applied electric pulse are presented in Figure 4 . The delay time $\tau_{\text {del }}$ defined as the time between the rising edge of the electric pulse and the start of system transmittance change, decreases from $6.6 \mathrm{~s}$ to $4.5 \mathrm{~s}$ in the range $3.7-4.1 \mathrm{~V}$ of control voltage. The turn-on time $\tau_{\mathrm{on}}$, defined as the time between rising edge of the electric pulse and the transmittance saturation, decreases from $29.3 \mathrm{~s}$ to $12.3 \mathrm{~s}$ in the same range of control voltage. Such dependences can be explained by the ion velocity rising resulted from the electric field increase. The turn-off time $\tau_{\text {off }}$ defined as the time between the falling edge of the electric pulse and the relaxation of transmittance to the initial zero level, increases from $7.2 \mathrm{~s}$ to $12.8 \mathrm{~s}$.

It should be noted that the ionic modification of the surface anchoring allows realizing other variants of the $\mathrm{LC}$ orientational transition, for example, the transition from the hybrid homeoplanar director configuration to the twisted-structure [18]. Apparently, this transition depends on neither the value nor the $\Delta \varepsilon$ sign. The use of this transition and optimizing the form of the control electric pulse [18] allow considerably reducing the optical response time (down to milliseconds) for the electro-optical LC cells operated by the ionic-surfactant method.

\section{Conclusion}

In conclusion, the orientational transitions induced by electrically controlled ionic modification of surface anchoring in nematics with small or zero dielectric anisotropy have been examined. It has been revealed that the external electric field causes the director reorientation from the homeotropic configuration into the hybrid one, which leads to switching of optical states of LC cell. This transformation depends on neither 
the sign nor the value of the LC dielectric anisotropy. That is, the same orientational transition can be realized for the nematics with $\Delta \varepsilon>0, \Delta \varepsilon<0$ and $\Delta \varepsilon=0$ by applying approximately the same value of control voltage. Thus, the ionic-surfactant method provides an opportunity to study electrically induced anchoring transitions and corresponding optical effects using all the variety of known nematics including LC with zero dielectric anisotropy.

\section{Disclosure statement}

No potential conflict of interest was reported by the authors.

\section{Funding}

This work was supported by Russian Foundation for Basic Research (RFBR) under Grants [15-02-06924 and 16-53-00073] and by the Siberian Branch of the Russian Academy of Sciences under Complex Program [II.2P 0358-2015-0010]. V.S.S. acknowledges financial support from RFBR under Grants [16-32-60036 and 16-3200164].

\section{References}

[1] Freedericksz VK, Zolina V. Forces causing the orientation of an anisotropic liquid. Trans Faraday Soc. 1933;29:919-930.

[2] Blinov LM. Structure and Properties of Liquid Crystals. Netherlands: Springer; 2011.

[3] Ryschenkow G, Kleman M. Surface defects and structural transitions in very low anchoring energy nematic thin film. J Chem Phys. 1976;64:404-412. doi:10.1063/1.431934.

[4] Volovik GE, Lavrentovich OD. Topological dynamics of defects: boojums in nematic drops. J Exp Theor Phys. 1983;58:1159-1166. 
[5] Kim JK, Araoka F, Jeong SM, et al. Bistable device using anchoring transition of nematic liquid crystals. Appl Phys Lett.2009;95:063505. doi:10.1063/1.3202781.

[6] Komitov L, Ichimura K, Strigazzi A. Light-induced anchoring transition in a 4,4'- disubstituted azobenzene nematic liquid crystal. Liq Cryst. 2000;27:51-55. doi:10.1080/026782900203209.

[7] Komitov L, Ruslim C, Matsuzawa Y, et al. Photoinduced anchoring transitions in a nematic doped with azo dyes. Liq Cryst. 2000;27:1011-1016. doi:10.1080/02678290050080733.

[8] Petrov AG, Durand G. Electric field transport of biphilic ions and anchoring transitions in nematic liquid crystals. Liq Cryst. 1994;17:543-554. doi:10.1080/02678299408036739.

[9] Jagemalm P, Komitov L, Barbero G. An electro-optic device based on fieldcontrolled anchoring of a nematic liquid crystal. Appl Phys Lett. 1998;73:16161618. doi:10.1063/1.122223.

[10] Fazio VSU, Komitov L. Alignment transition in a nematic liquid crystal due to field-induced breaking of anchoring. Europhys Lett. 1999;46:38-42. doi:10.1209/epl/11999-00559-8.

[11] Komitov L, Helgee B, Felix J, et al. Electrically commanded surfaces for nematic liquid crystal displays. Appl Phys Lett. 2005;86:023502. doi:10.1063/1.1849844.

[12] Zyryanov VY, Krakhalev MN, Prishchepa OO, et al. Orientational structure transformations caused by the electric-field-induced ionic modification of the interface in nematic droplets. J Exp Theor Phys Lett. 2007;86:383-388. doi:10.1134/S0021364007180087.

[13] Zyryanov VY, Krakhalev MN, Prishchepa OO, et al. Inverse regime of ionic modification of surface anchoring in nematic droplets. J Exp Theor Phys Lett. 2008;88:597-601. doi:10.1134/S002136400821011X.

[14] Zyryanov VY, Krakhalev MN, Prishchepa OO. Texture transformation in nematic droplets caused by ionic modification of boundary conditions. Mol Cryst Liq Cryst. 2008;489:273/[599]-279/[605]. doi:10.1080/15421400802219189. 
[15] Krakhalev MN, Prishchepa OO, Zyryanov VY. Inverse mode of ion-surfactant method of director reorientation inside nematic droplets. Mol Cryst Liq Cryst. 2009;512:152/[1998]-157/[2003]. doi:10.1080/15421400903050814.

[16] Sutormin VS, Krakhalev MN, Prishchepa OO, et al. Electrically controlled local Frédericksz transition in a layer of a nematic liquid crystal. J Exp Theor Phys Lett. 2012;96:511-516. doi:10.1134/S0021364012200131.

[17] Sutormin VS, Krakhalev MN, Zyryanov VY. The dynamics of the response of an electro-optic cell based on a nematic layer with controlled surface anchoring. Tech Phys Lett. 2013;39:583-586. doi:10.1134/S1063785013070134.

[18] Sutormin VS, Krakhalev MN, Prishchepa OO, et al. Electro-optical response of an ionic-surfactant-doped nematic cell with homeoplanar-twisted configuration transition. Opt Mater Express. 2014;4:810-815. doi:10.1364/OME.4.000810.

[19] Klingbiel RT, Genova DJ, Bucher HK. The Temperature Dependence of the Dielectric and Conductivity Anisotropies of Several Liquid Crystalline Materials. Mol Cryst Liq Cryst. 1974;27:1-21. doi:10.1080/15421407408083116.

[20] Bradshaw MJ, Raynes EP, Bunning JD, et al. The Frank constants of some nematic liquid crystals. J Physique 1985;46:1513-1520. doi:10.1051/jphys:019850046090151300.

[21] Ohtsu M, Akahane T, Tako T. Birefringence of n-Type Nematic Liquid Crystals Due to Electrically Induced Deformations of Vertical Alignment. Jap J Appl Phys. 1974;13:621-629. doi:10.1143/JJAP.13.621. 
Figure 1. The Frederiks effect in LC cells based on the nematics with positive (a), negative (b) and zero (c) dielectric anisotropy induced by the electric field. The initial LC orientations are shown in the left column. The right column represents LC orientations under the action of the ac electric field applied perpendicularly to the substrates.

Figure 2. The scheme of the LC orientational transition induced by the electrically controlled ionic modification of surface anchoring. Under the dc electric field, LC is reoriented from the initial homeotropic configuration (a) into the hybrid one (b) independently on the sign and value of the dielectric anisotropy $\Delta \varepsilon$ of the used nematic.

Figure 3. Optical textures of the LC cell based on the MBBA-5CB mixture $(\Delta \varepsilon=0)$ doped with CTAB in the initial state (a) and under the dc electric field $4 \mathrm{~V}$ (b); the square-wave optical response of the LC cell (the top curve) placed between the crossed polarizers and electric $4 \mathrm{~V}$ pulse (the bottom curve) (c). The polarizer directions are shown by the double arrows. $\mathbf{R}_{1}$ and $\mathbf{R}_{2}$ are rubbing directions of the top and bottom substrates, respectively.

Figure 4 . Delay time $\tau_{\text {del }}$ (left axis), turn-on time $\tau_{\text {on }}$ and turn-off time $\tau_{\text {off }}$ (right axis) depending on the control voltage for the LC cell based on the MBBA-5CB mixture $(\Delta \varepsilon=0)$ doped with CTAB. 


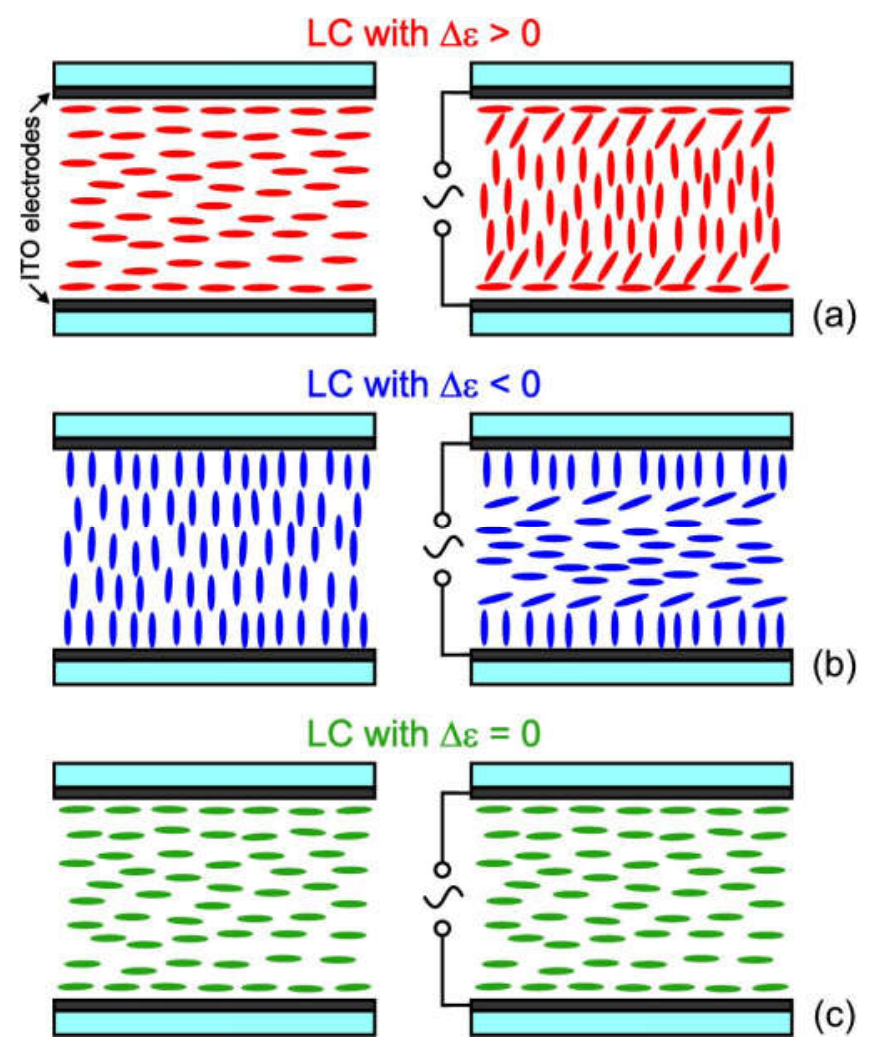

Figure 1. The Frederiks effect in LC cells based on the nematics with positive (a), negative (b) and zero (c) dielectric anisotropy induced by the electric field. The initial LC orientations are shown in the left column. The right column represents LC orientations under the action of the ac electric field applied perpendicularly to the substrates. 


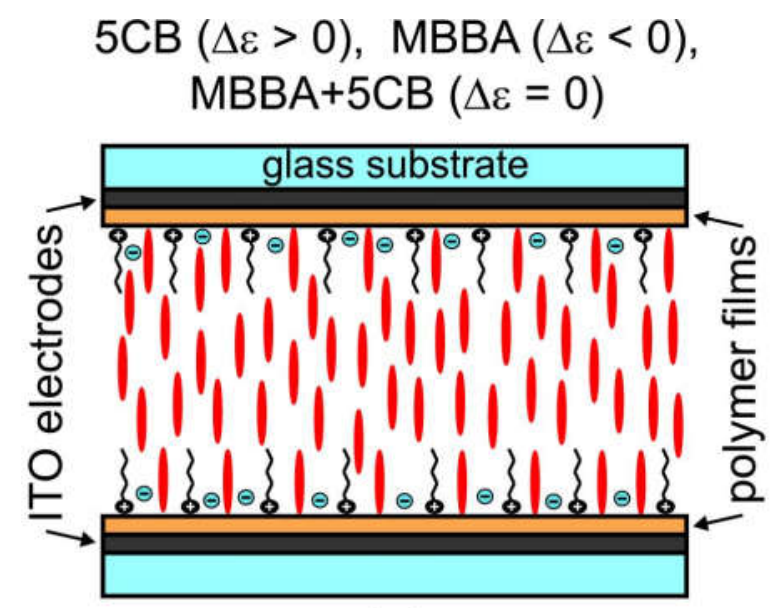

(a)

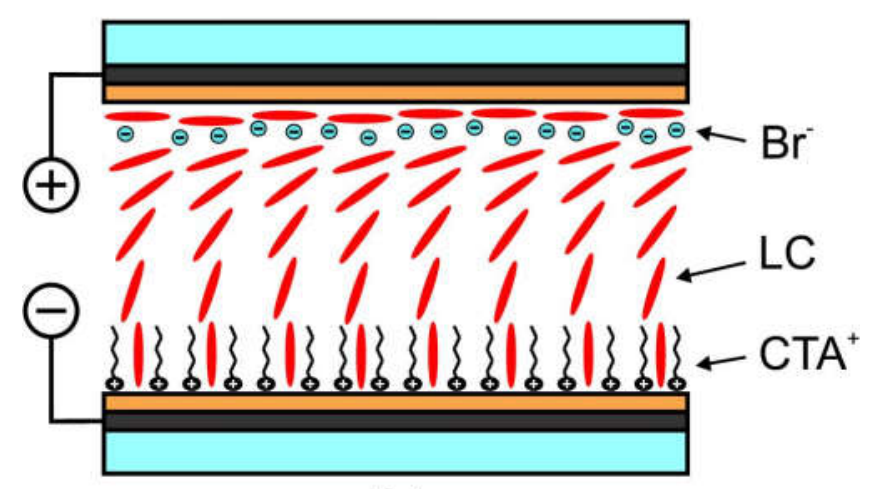

(b)

Figure 2. The scheme of the LC orientational transition induced by the electrically controlled ionic modification of surface anchoring. Under the dc electric field, LC is reoriented from the initial homeotropic configuration (a) into the hybrid one (b) independently on the sign and value of the dielectric anisotropy $\Delta \varepsilon$ of the used nematic. 


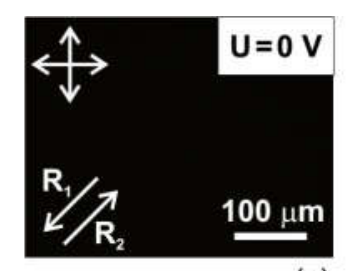

(a)

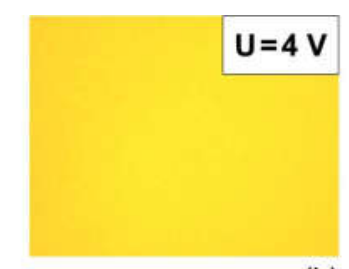

(b)

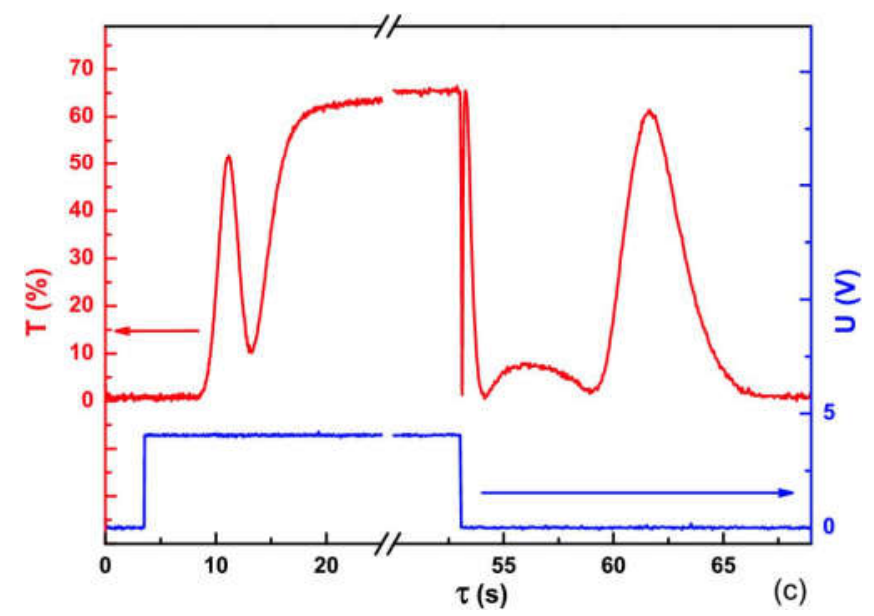

Figure 3. Optical textures of the LC cell based on the MBBA-5CB mixture $(\Delta \varepsilon=0)$ doped with CTAB in the initial state (a) and under the dc electric field $4 \mathrm{~V}$ (b); the square-wave optical response of the LC cell (the top curve) placed between the crossed polarizers and electric $4 \mathrm{~V}$ pulse (the bottom curve) (c). The polarizer directions are shown by the double arrows. $\mathbf{R}_{1}$ and $\mathbf{R}_{2}$ are rubbing directions of the top and bottom substrates, respectively.

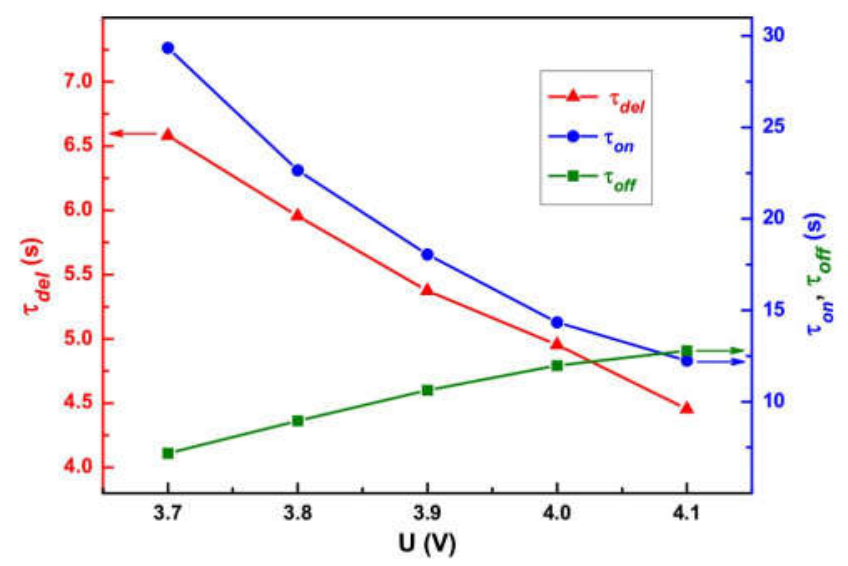

Figure 4 . Delay time $\tau_{\text {del }}$ (left axis), turn-on time $\tau_{\text {on }}$ and turn-off time $\tau_{\text {off }}$ (right axis) depending on the control voltage for the LC cell based on the MBBA-5CB mixture $(\Delta \varepsilon=0)$ doped with CTAB. 